\title{
Improvement of Bit Rate Using M-ary Chaotic Pulse Position Modulation
}

\author{
Nguyen Xuan Quyen, Vu Van Yem, Thang Manh Hoang \\ School of Electronics and Telecommunications, Hanoi University of Science and Technology, 1 Dai Co \\ Viet, Hanoi, Vietnam
}

Correspondence: Vu Van Yem, yemvv-fet@mail.hut.edu.vn

Manuscript communication: received 29 October 2011, revised 26 November 2011

\begin{abstract}
Recent studies have pointed out that Chaotic Pulse Position Modulation (CPPM) is a very promising method for improving privacy and security in chaos-based digital communication. Especially, CPPM provides better performance than other chaotic modulation methods in noise- and distortion-affected environments. In this paper we present our development of a robust method named M-ary CPPM which is based on the combination of the conventional CPPM and multi-symbol modulation in order to improve the transmission bit rate. The $M$-ary CPPM signal has a pulse train format in which each pulse is a symbol and the chaotically-varied inter-pulse time interval conveys the binary information of $k$ bits $\left(M=2^{k}\right)$. The analysis and development of modulation and demodulation schemes are presented in detail. Theoretical evaluation of Bit-Error-Rate (BER) performance in the presence of additive white Gaussian noise (AWGN) and the use of AWGN filtering is also provided. The chaotic behavior of the $M$-ary CPPM is investigated with the variation of modulation parameters. In order to verify the performance of the proposed schemes, numerical simulations were carried out in Simulink and comparison between simulation and theoretical results is reported.
\end{abstract}

Keywords- chaotic modulation, chaotic pulse position modulation, CPPM, M-ary modulation, chaos-based digital communication, ultra-wide band, tent map.

\section{INTRODUCTION}

In recent years, chaotic signals and chaotic systems have been investigated in various research fields such as physics, biology, chemistry, and engineering [1]. Chaosbased digital communication has received significant attention [2] due to its potential in improving the privacy and security of information [3]. Many chaosbased modulation methods have been proposed using different modulation schemes [3, 4]. Due to their type of demodulation, these schemes can be classified as coherent or non-coherent detection. Each method has its own advantages and disadvantages but most of them use the chaotic carrier created by a chaotic dynamical system to convey information, so they are sensitive to distortion and noise that can strongly affect the synchronization [5-7] and cause errors in recovering information.

A robust modulation method called Chaotic Pulse Position Modulation was proposed in [8, 9] to overcome the major drawbacks mentioned above. Since binary information is only modulated onto the inter-pulse intervals, not the phase, frequency or amplitude of the pulses, the impact of distortion and noise on the pulse shape does not seriously affect the synchronization process. In CPPM, only one bit is decoded at the moment of a received pulse, that leads to low transmission rate. To improve the bit rate, we propose a modified modulation method which is the combination of the conventional CPPM and multi-symbol modulation, named M-ary CPPM. The proposed method uses each chaoticallyvaried inter-pulse interval as a carrier to convey the binary information of $k$ bits, where each pulse is considered as a symbol. It means that a volume of $k$ bits is decoded for each instance of received pulses, thus the bit rate will increase significantly. Like the CPPM method, the $M$-ary CPPM also performs well in distortion- and noise-affected channels and achieves high levels of information privacy as well as automatic synchronization without the need of specific handshaking protocols [10]. Especially, this method is effective for direct transmission of ultra-narrow and lowpower pulses and therefore suitable for ultra-wide band (UWB) communication [11, 12].

Tent map is a discrete-time and one-dimension nonlinear function with the piecewise-linear I/O characteristic curve [13], used for generating chaotic values which look like true random numbers [14]. In chaosbased communications, the tent map is proposed for application in chaotic modulation [15] with such advantages as the simplified calculation and the robust regimes of chaos generation for rather broad ranges of modulations parameters. Therefore, the utilization of tent map for the $M$-ary CPPM is investigated with the variation of modulation parameters. This is very important for the design to guarantee the chaotic behavior of the method. 


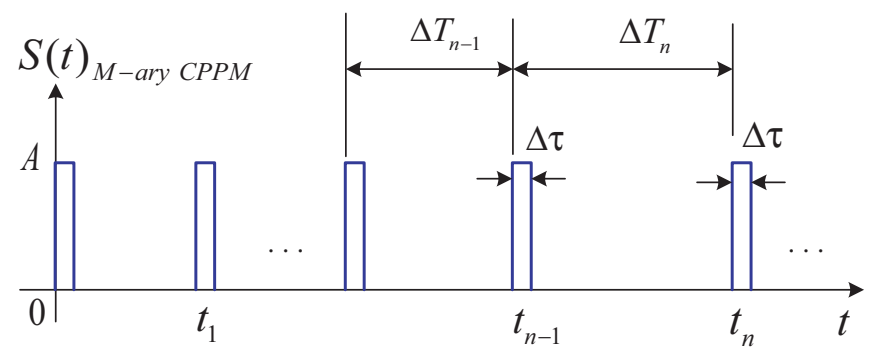

Figure 1. M-ary CPPM signal.

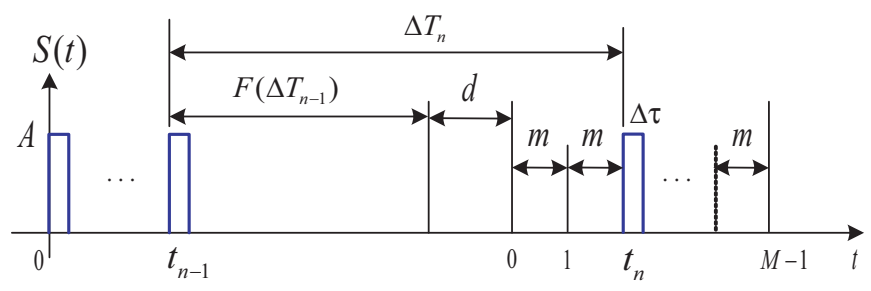

Figure 2. Time interval $\Delta T_{n}$ with $M$ symbols.

The rest of this paper is organized as follows. The principle of the M-ary CPPM is described in Section 2. Section 3 presents our analysis of the schemes for modulator and demodulator in detail. In Section 4, BER performance of the method is evaluated theoretically based on a recursive algorithm. Section 5 investigates the chaotic behavior of the $M$-ary CPPM with tent map. In order to verify the performance of the proposed schemes, a numerical simulation is implemented in Simulink and its results in the time domain as well as the resulted BER performance are presented in Section 6. Finally, our conclusions are given in Section 7.

\section{Description}

In this section, we describe the principles of the $M$-ary CPPM method. $M$-ary CPPM signal is considered as a chaotic pulse train which is illustrated in Figure 1 and can be expressed as follows:

$$
S(t)_{M \text {-ary CPPM }}=\sum_{n=0}^{\infty} A\left[u\left(t-t_{n}\right)-u\left(t-t_{n}-\Delta \tau\right)\right],
$$

where $u(t)$ is the unit-step function, $A$ and $\Delta \tau$ are the amplitude and width of the pulses, respectively. The $n^{\text {th }}$ pulse is generated at time $t_{n}=t_{0}+\sum_{n=0}^{n} \Delta T_{n}$ with $\Delta T_{n}$ being the time interval between the $n$-th and $(n-1)$-th pulses. This interval is iteratively produced by a nonlinear transformation $F\left(\Delta T_{n-1}\right)$. By choosing a nonlinear function $F(\cdot)$ with appropriate modulation parameters, $\Delta T_{n}$ will vary in a chaotic way and has a random-like behavior. The interval $\Delta T_{n}$ with $M$ symbols is shown in Figure 2.

In the $M$-ary CPPM modulator, the binary information is modulated onto inter-pulse interval as follows:

$$
\Delta T_{n}=F\left(\Delta T_{n-1}\right)+d+m S_{n},
$$

where $S_{n}$ is the reference value of the $n^{\text {th }}$ symbol. The source data is divided into $k$-bit groups which are then mapped to equivalent symbols. The value of $S_{n}$ varies

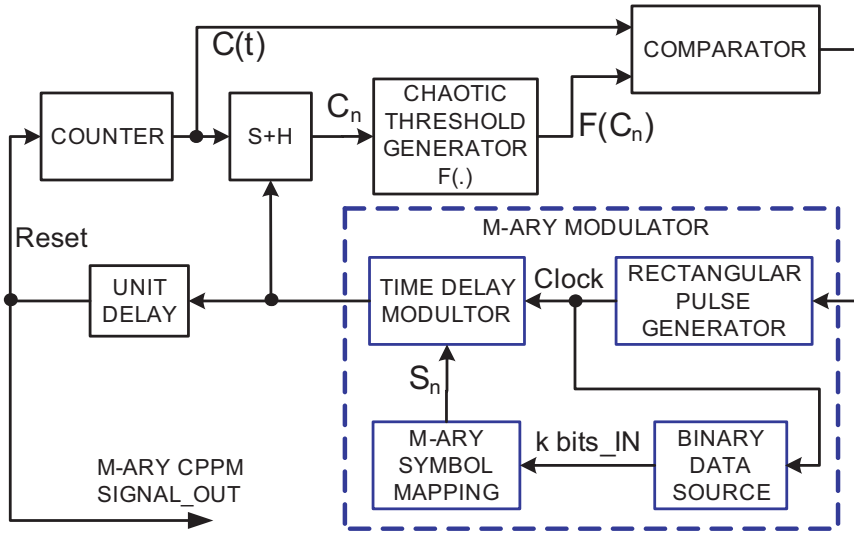

Figure 3. Scheme for the $M$-ary CPPM modulator.

from 0 to $M-1$ with $M$ being the number of symbols $\left(M=2^{k}\right)$. The parameter $d$ is a constant time delay that is inserted to guarantee the synchronization of the system. The parameter $m$ is the modulation depth and it directly affects the bit error rate as well as the optimization of the transmission speed.

In the $M$-ary CPPM demodulator, the parameters $d$, $m$ and $F(\cdot)$ are the same as in the modulator. Each received pulse carries information of a symbol and guides the synchronization process. When the synchronization state of the system is maintained, suppose that the demodulator receives the interval $\Delta T_{n-1}$ correctly, it easily computes $S_{n}$ by the following equation:

$$
S_{n}=\frac{\Delta T_{n}-F\left(\Delta T_{n-1}\right)-d}{m} .
$$

From the value of $S_{n}$, the $n^{\text {th }}$ symbol is found and remapped to $k$ bits, thus the binary data is recovered.

Similar to the CPPM, the M-ary CPPM method also has a chaotic pulse train format, thus its system automatically acquires synchronization as in a CPPMbased system. The demodulator only needs to correctly detect two consecutive pulses in order to re-establish the synchronization state.

\section{Modulator and Demodulator}

In this section, the operation of an $M$-ary CPPM-based system is examined through the design and analysis of the schemes for modulator and demodulator.

\subsection{Modulator}

The scheme for an M-ary CPPM modulator is shown in Figure 3 and its signals are illustrated in Figure 4. The Counter operates in free running mode to produce a linearly increasing value $C(t)=K t$ at its output, where the time $t$ is the duration from the reset time and $K$ is the count step (the difference value between two consecutive samples). At the time $t_{n-1}$, the counter is reset, i.e., $C\left(t_{n-1}\right)=0$, and then it starts to count immediately. The output signal $C(t)$ is put into a Comparator to compare with a threshold value $F\left(C_{n-1}\right)$ which is produced at the output of the Chaotic Threshold Generator $F(\cdot)$. Here, $C_{n-1}$ is the output 


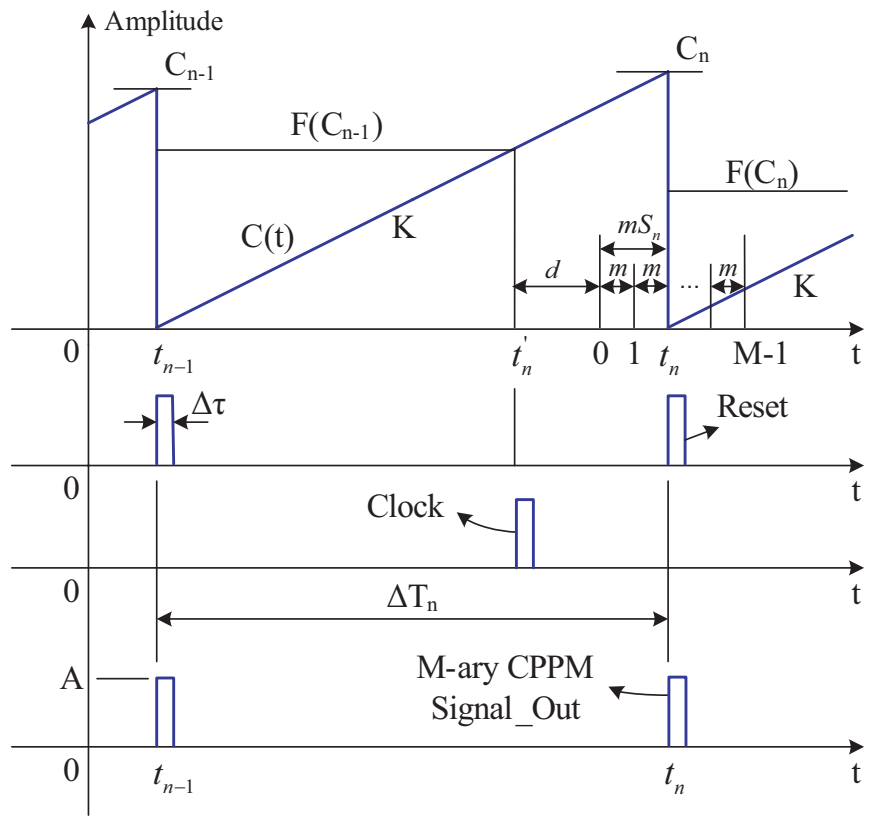

Figure 4. Chaotic signals of the $M$-ary CPPM modulator.

value of the counter which was acquired and saved by a Sample and Hold $(\mathrm{S}+\mathrm{H})$ block from the previous iteration before the reset time. When the output of the counter $C(t)$ reaches the threshold value $F\left(C_{n-1}\right)$, the output of the comparator triggers a Rectangular Pulse Generator to produce a clock pulse at the time $t_{n}^{\prime}=t_{n-1}+F\left(C_{n-1}\right) / K$. Then, this clock pulse triggers a Binary Data Source to map a $k$-bit number to a new symbol $S_{n}$ at the $M$-ary Symbol Mapping block. At the Time Delay Modulator, depending on the input value $S_{n}$, the clock pulse will be delayed by a time duration $d+m S_{n}$. Therefore, the modulated pulse is generated at the time $t_{n}=t_{n}^{\prime}+d+m S_{n}$. After that, the modulated pulse will trigger the $\mathrm{S}+\mathrm{H}$ block and reset the counter. A new iteration starts and the next symbol will be modulated in the same way. The reset pulses are the same pulses of the output $M$-ary CPPM signal. From the above equations, the time interval between the $n^{\text {th }}$ and $(n-1)^{\text {th }}$ pulses is determined by

$$
\begin{aligned}
\Delta T_{n}=t_{n}-t_{n-1} & =\frac{F\left(C_{n-1}\right)}{K}+d+m S_{n} \\
& =\frac{F\left(K \Delta T_{n-1}\right)}{K}+d+m S_{n}
\end{aligned}
$$

With $K, d$ and $m$ being constants, Eq. (4) points out that the time interval $\Delta T_{n}$ depends on the reference value $S_{n}$ and the chaotic threshold $F\left(K \Delta T_{n-1}\right)$.

\subsection{Demodulator}

The scheme and signals of the $M$-ary CPPM demodulator are shown in Figures 5 and 6, respectively. The Counter, the Chaotic Threshold Generator $F(\cdot)$, the $\mathrm{S}+\mathrm{H}$ and the Rectangular Pulse Generator blocks are the same as in the modulator. At the input of the demodulator, the $M$-ary CPPM signal is put into a Threshold Detector. When this signal exceeds the threshold value, a rectangular pulse is generated by the

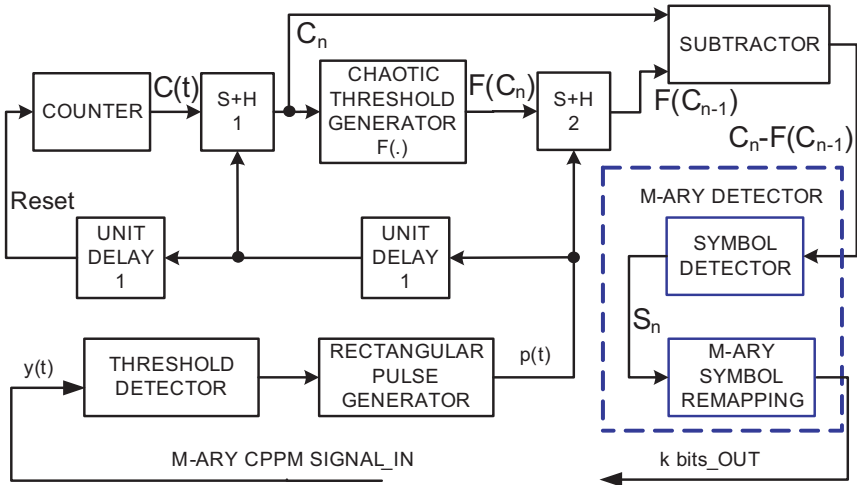

Figure 5. Scheme of the M-ary CPPM Demodulator.

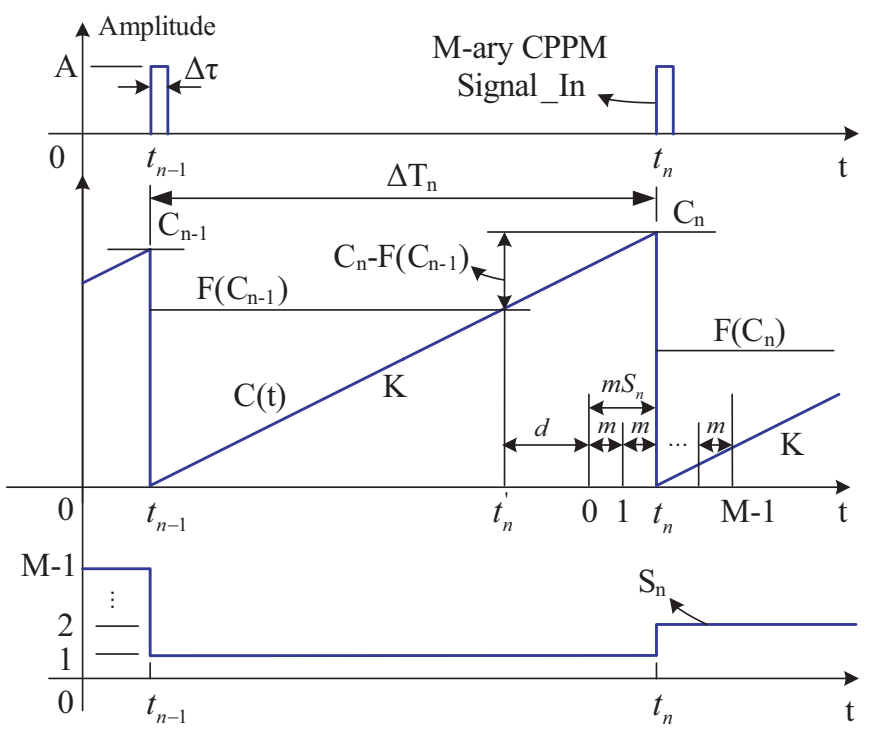

Figure 6. Chaotic signals of the $M$-ary CPPM demodulator

Rectangular Pulse Generator. This pulse at first triggers the block $\mathrm{S}+\mathrm{H} 2$ to save the value $F\left(C_{n-1}\right)$ from the $F(\cdot)$ output, then triggers the block $\mathrm{S}+\mathrm{H} 1$ to store the value of the counter $C_{n}$ at that time, and then resets the counter. The two values saved by the $\mathrm{S}+\mathrm{H}$ blocks are put into a Subtractor to produce a difference value at the output. From Eq. (4), it is determined by

$$
C_{n}-F\left(C_{n-1}\right)=K \Delta T_{n}-F\left(C_{n-1}\right)=K\left(d+m S_{n}\right) .
$$

After that, the difference value is put into a Symbol Detector to decode the reference value $S_{n}$ of the symbol as follows:

$$
S_{n}=\frac{\frac{C_{n}-F\left(C_{n-1}\right)}{K}-d}{m} .
$$

Depending on the value $S_{n}$, the $n^{\text {th }}$ symbol is identified and $k$ bits are recovered at the output of the $M$-ary Symbol Re-mapping block.

\section{Theoretical Evaluation of BER Performance}

An analytical method to evaluate the CPPM error probability was reported in [8], and it is used for estimating the BER of the M-ary CPPM in this research. For 


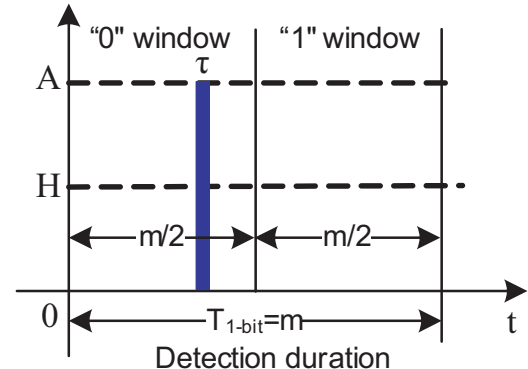

Figure 7. Detection windows of the Binary-CPPM.

simplicity, let's consider the input signal of the $M$ ary CPPM demodulator, $y(t)$, as the filtered signal of the sum of the transmitted M-ary CPPM signal and the AWGN. In the threshold detector, when the signal $y(t)$ exceeds a threshold value $H$, the rectangular pulse generator is triggered to generate a rectangular pulse, $p(t)$, with an amplitude $A$ at its output. This pulse will be used to recover the original data bits. Firstly, we consider a simple case with the Binary CPPM $(M=2)$. In Figure 7, the detection duration with the width $m$ is divided into the " 0 " window and the " 1 " window, both have a same width, where $m$ is the modulation depth and $\tau$ is the fundamental sampling period of the system. Assume that the demodulator maintains the synchronization at all times. Bit error will occur when the pulse $p(t)$ falls into an unexpected window. It means that the pulse $p(t)$ of a transmitted bit " 0 " falls into the "1" window and vice versa. From [8], the evaluation result of the BER is determined by

$$
\begin{aligned}
& \text { BER }_{\text {Binary }} \text { СPMM }=p_{1} P_{0 / 1}+\left(1-p_{1}\right) P_{1 / 0} \\
& =p_{1}\left[1-\left[\frac{1}{2}\left(1+\operatorname{erf}\left(h \sqrt{E_{b} / N_{o}}\right)\right)\right]^{\frac{m}{2 \tau}}\right] \\
& +\left(1-p_{1}\right) \times\left[\left[\frac{1}{2}\left(1+\operatorname{erf}\left(h \sqrt{E_{b} / N_{o}}\right)\right)\right]^{\frac{m}{2 \tau}-1}\right. \\
& \left.\times \frac{1}{2} \operatorname{erfc}\left((1-h) \sqrt{E_{b} / N_{o}}\right)\right]
\end{aligned}
$$

where $P_{0 / 1}$ and $P_{1 / 0}$ are the error probabilities that a " 0 " is detected when an " 1 " is transmitted and vice versa; $p_{1}$ is the proportion of " 1 " bits in the entire data stream; and $h=\frac{H}{A}$. In the case that both $p_{1}$ and $h$ are equal to $\frac{1}{2}$, the expression for the BER can be written in a shorter form as

$$
\begin{aligned}
& \text { BER }_{\text {Binary CPPM }}=\frac{1}{2}\left[1-\operatorname{erf}\left(\frac{1}{2} \sqrt{E_{b} / N_{o}}\right)\right. \\
& \left.\times\left[\frac{1}{2}\left(1+\operatorname{erf}\left(\frac{1}{2} \sqrt{E_{b} / N_{o}}\right)\right)\right]^{\frac{m}{2 \tau}-1}\right] .
\end{aligned}
$$

Secondly, from the above results, we consider the general case for BER evaluation of the $M$-ary CPPM ( $k$ bits) with the detection duration illustrated in Figure 8. The pulse $p(t)$ can appear in the detection duration with the width $T_{k \text {-bit }}=(M-1) m$. This duration is divided into two windows having the same width of $T_{k \text {-bit }} / 2$. In these, the left window contains symbols with the " 0 " prefix and the right one contains symbols with the

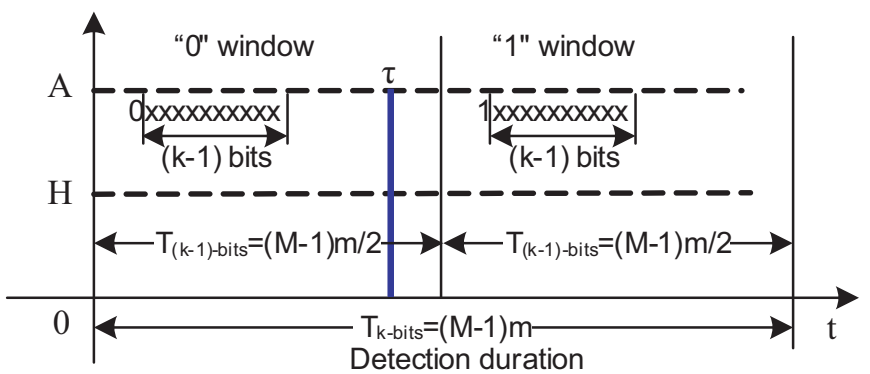

Figure 8. Detection windows of the $M$-ary CPPM.

" 1 " prefix. Therefore, the error probability of the first detected bit, $P_{1 s t-b i t}$, is also the error probability of the pulse in the detection duration $T_{k \text {-bit }}$. From Eq. (8), we have

$$
\begin{aligned}
P_{1 s t \text {-bit }}= & T_{k \text {-bit }}=\frac{1}{2}\left[1-\operatorname{erf}\left(\frac{1}{2} \sqrt{E_{b} / N_{o}}\right)\right. \\
& \left.\times\left[\frac{1}{2}\left(1+\operatorname{erf}\left(\frac{1}{2} \sqrt{E_{b} / N_{o}}\right)\right)\right]^{\frac{T_{k-\text { bit }}^{2 \tau}}{2 \tau}-1}\right] .
\end{aligned}
$$

In case the first bit is recovered correctly, the $M$-ary CPPM error probability is then the error probability of recovering the rest $(k-1)$ bits with the detection duration of $T_{(k-1) \text {-bit }}=(M-1) m / 2$. Therefore, the $M$-ary CPPM error probability for transmitting a $k$-bit sequence can be inferred from the following recursive equation:

$$
\begin{aligned}
& \mathrm{BER}_{M \text {-ary CPPM }} \equiv \mathrm{BER}_{k \text {-bit }} \\
& =P_{1 s t \text {-bit }}+\left(1-P_{1 s t-\text { bit }}\right) \mathrm{BER}_{(k-1) \text {-bit }} \\
& =P_{T_{k-\text { bit }}}+\left(1-P_{T_{k \text {-bit }}}\right) \mathrm{BER}_{(k-1) \text {-bit }} \\
& =\mathrm{BER}_{(k-1) \text {-bit }}+P_{T_{k-\text { bit }}}\left(1-\mathrm{BER}_{(k-1) \text {-bit }}\right),
\end{aligned}
$$

with the initial equation being

$$
\begin{aligned}
& \mathrm{BER}_{4 \text {-ary } \mathrm{CPPM}} \equiv \mathrm{BER}_{2 \text {-bit }} \\
& =\mathrm{BER}_{1 \text {-bit }}+P_{T_{2} \text {-bit }}\left(1-\mathrm{BER}_{1 \text {-bit }}\right),
\end{aligned}
$$

where $\mathrm{BER}_{1 \text {-bit }} \equiv \mathrm{BER}_{\text {Binary CPPM }}$ is determined by Eq. (8), $P_{T_{2-\text { bit }}}$ is determined by Eq. (9) with the detection duration $T_{2 \text {-bit }}=3 \mathrm{~m}$.

BER evaluation results of 2-, 4-, 8- and 16-ary CPPM according to Eq. (10) and Eq. (11) are shown in Figure 13 (see the blue lines), in which the modulation depth is chosen by $m=10 \tau$.

\section{M-ARy CPPM with Tent MaP}

The conventional tent map is iteratively generated through a transformation function $F(\cdot):(0,1) \rightarrow(0,1)$ as given by

$$
\begin{aligned}
x_{n+1} & =F\left(x_{n}\right)=F^{n}\left(x_{0}\right) \\
& = \begin{cases}a x_{n}, & 0<x_{n} \leq 0.5 \\
a\left(1-x_{n}\right), & 0.5<x_{n}<1\end{cases}
\end{aligned}
$$

In this equation, $n$ represents the number of iterations, $x_{0}$ is the initial value, $x_{n}$ is the output value at the $n$-th step, and parameter $a$ controls the state of the map. 


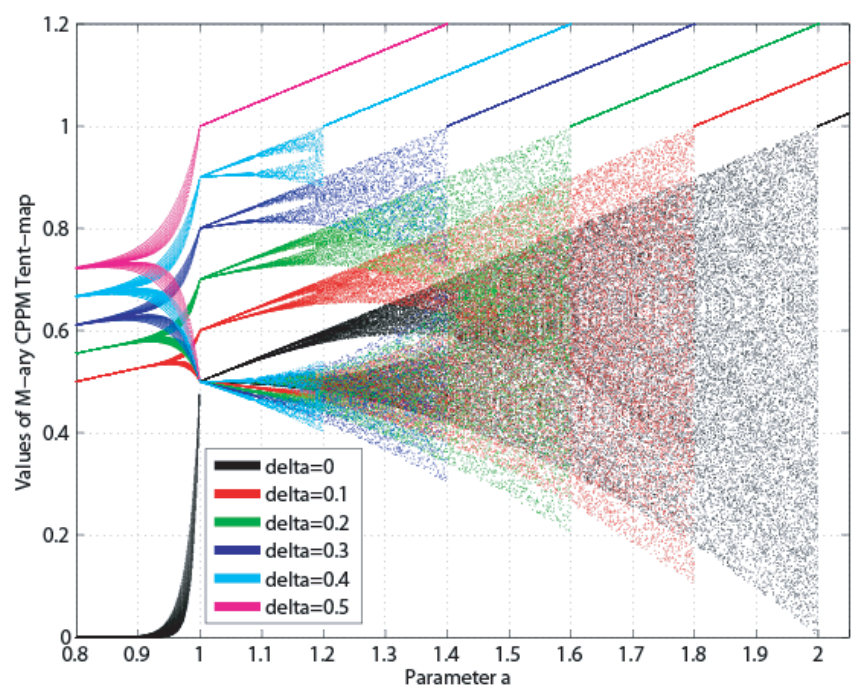

Figure 9. Bifurcation diagram of the $M$-ary CPPM tent map with variations of parameters $a$ and $\delta$ (delta).

In the $M$-ary CPPM, the time interval between the $n$ th and $(n-1)$-th pulses is expressed by Eq. (4) which can be converted to the following:

$$
\Delta T_{n}=\frac{F\left(K \Delta T_{n-1}\right)}{K}+d+m S_{n}
$$

or

$$
X_{n}=F\left(X_{n-1}\right)+d^{\prime}+m^{\prime} S_{n}
$$

where $X_{n}=K \Delta T_{n}, X_{n-1}=K \Delta T_{n-1}, d^{\prime}=K d$, and $m^{\prime}=$ $K m$. From Eq. (12) and Eq. (14), the tent map for the $M$ ary CPPM, called the M-ary CPPM tent map, is derived as

$$
\begin{aligned}
X_{n+1} & =F\left(X_{n}\right) \\
& = \begin{cases}a X_{n}+d^{\prime}+m^{\prime} S_{n}, & 0<X_{n} \leq 0.5 \\
a\left(1-X_{n}\right)+d^{\prime}+m^{\prime} S_{n}, & 0.5<X_{n}<1\end{cases}
\end{aligned}
$$

In the modulation process, the value of the symbol $S_{n+1}$ varies from 0 to $M-1$, thus the value of the parameter $\delta=d^{\prime}+m^{\prime} S_{n}$ varies from $d^{\prime}$ to $d^{\prime}+m^{\prime}(M-1)$. This variation of $\delta$ makes the chaotic behavior of the $M$ ary CPPM tent map dependent not only on the control parameter $a$ but also on the parameters $d^{\prime}, m^{\prime}$ and $M$. Figure 9 presents the bifurcation diagram of the $M$-ary CPPM tent map, which clearly shows the dependence of the chaotic behavior on the parameters $a$ and $\delta$. In the figure, $\delta=0$ is the case of the conventional tent map. The more the value of $\delta$ increases, the smaller the chaotic area becomes, and when $\delta \geq 0.5$ the chaotic area disappears. In practice, the parameters $d^{\prime}, m^{\prime}$ and $M$ are determined first based on design specifications such as bit rate and BER. After that, the control parameter $a$ is chosen to guarantee the chaotic behavior of the system. For example, suppose we have a 4-ary CPPM tent map $(M=4)$ with given parameters $d^{\prime}=0.075$ and $m^{\prime}=0.075$, the maximum value of $\delta$ is then determined by

$$
\delta_{\max }=d^{\prime}+m^{\prime}(M-1)=0.075+0.075 \times 3=0.3
$$
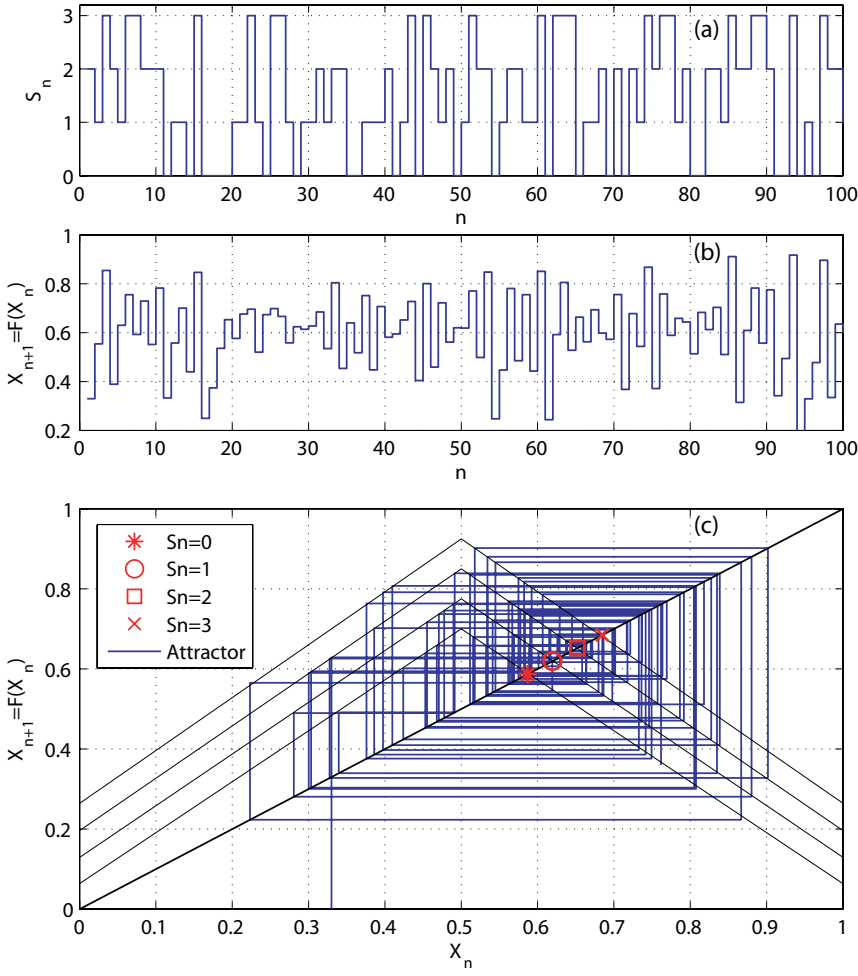

Figure 10. The 4-ary CPPM tent map with parameters $a=1.3$, $x_{0}=0.1, d^{\prime}=0.075$, and $m^{\prime}=0.075$. (a) and (b): reference values of the symbols and output values of the map, respectively, for 100 iterations; (c): attractor diagram with four fixed points.

From the bifurcation diagram, it is drawn that the control parameter $a$ must be chosen such that $1<a<1.4$ in order to obtain a chaotic behavior for the system.

Simulation results for the above-mentioned 4-ary CPPM tent map in the first 100 iterations with $X_{0}=0.1$ and $a=1.3$ are shown in Figure 10. It is shown that the output values of the map (Figure 10b) vary chaotically but also convey the information of the symbol values (presented in Figure 10a). Besides, the chaotic behavior of the map is displayed by the attractor diagram in Figure 10c that have four fixed points (red markers) corresponding to four values of the symbols.

\section{Numerical Simulation And Results}

In order to verify the performance of the presented $M$ ary CPPM modulation and demodulation schemes, a numerical simulation of the system with an AWGN channel and without parameter mismatch is implemented in Simulink. It means that a same set of values for the modulation parameters is used in both modulator and demodulator. Simulations are performed for $M=2,4,8$, and 16 . The values of other modulation parameters are chosen as follows: $K=0.002 / \tau, d=10 \tau$, $m=10 \tau$, and $\Delta T_{0}=50 \tau$, with $\tau$ being the fundamental sampling period of the system. From Eq. (14) and Figure 9, the parameters of the M-ary CPPM tent map 
Table I

Transmission Rate of 2, 4, 8, 16-Ary CPPM System Per $10^{6}$ Fundamental Sample Time

\begin{tabular}{|c||c||c|}
\hline $\begin{array}{c}\text { Number of } \\
\text { symbols M }\end{array}$ & $\begin{array}{c}\text { Number of } \\
\text { transmitted pulses }\end{array}$ & $\begin{array}{c}\text { Number of } \\
\text { transmitted bits }\end{array}$ \\
\hline 2 & 3749 & 3749 \\
\hline 4 & 3387 & 6774 \\
\hline 8 & 3328 & 9984 \\
\hline 16 & 3284 & 13136 \\
\hline
\end{tabular}

are then derived as:

$$
\begin{aligned}
X_{0} & =K \Delta T_{0}=\left(\frac{0.002}{\tau} \times 50 \tau\right)=0.1 \\
d^{\prime} & =K d=\left(\frac{0.002}{\tau} \times 10 \tau\right)=0.02 \\
m^{\prime} & =K m=\left(\frac{0.002}{\tau} \times 10 \tau\right)=0.02
\end{aligned}
$$

Therefore,

$$
\begin{aligned}
& \delta_{\max }(M=2,4,8,16)=\delta_{\max }(M=16) \\
& \quad=d^{\prime}+m^{\prime}(16-1)=0.02+0.02 \times 15=0.32
\end{aligned}
$$

Then, the value for $a$ is selected as $a=1.3$ to guarantee the chaotic behavior of the simulated system for all four cases of $M(2,4,8$, and 16).

\subsection{Chaotic Signals in Time Domain}

An 8-ary CPPM system with an AWGN channel is simulated, in which $E_{b} / N_{o}=20 \mathrm{~dB}$ to ensure a good transmission line. Simulated chaotic signals in the 8-ary CPPM modulator and demodulator, within the duration from the starting time 0 to $5000 \tau$, are shown in Figure 11 and Figure 12, respectively. We can observe that the time interval $\Delta T_{n}$ varies chaotically in the range between $150 \tau$ and $500 \tau$. The widths of the reset, clock and $M$-ary CPPM pulses are the same and equal to $20 \tau$. At each time instant, $S_{n}$ receives only one of the eight values, $0,1,2,3,4,5,6$ or 7 , corresponding to one of the eight symbols, $000,001,010,011,100,101,110$ or 111 , respectively.

When the synchronization state of the system is maintained, the signals recovered by the demodulator exactly match their corresponding signals in the modulator. It verifies that the proposed schemes for the $M$ ary CPPM method are suitable and feasible.

\subsection{Improvement of Bit-Rate Performance}

Table I compares simulation results of transmission rates between the 2-, 4-, 8- and 16-ary CPPM schemes with a time duration of $10^{6} \tau$ and $E_{b} / N_{o}=20 \mathrm{~dB}$ for all simulations. These results show that the numbers of transmitted pulses (i.e., the symbol rates) of the 4-, 8and 16-ary CPPM schemes are only slightly lower than that of the binary CPPM scheme, but their numbers of transmitted bits (i.e., the bit rates) are much higher than the binary CPPM bit rate. That means a significant improvement in bit-rate performance of the $M$-ary CPPM compared to the conventional CPPM. According to these results, if the processing speed of modulator
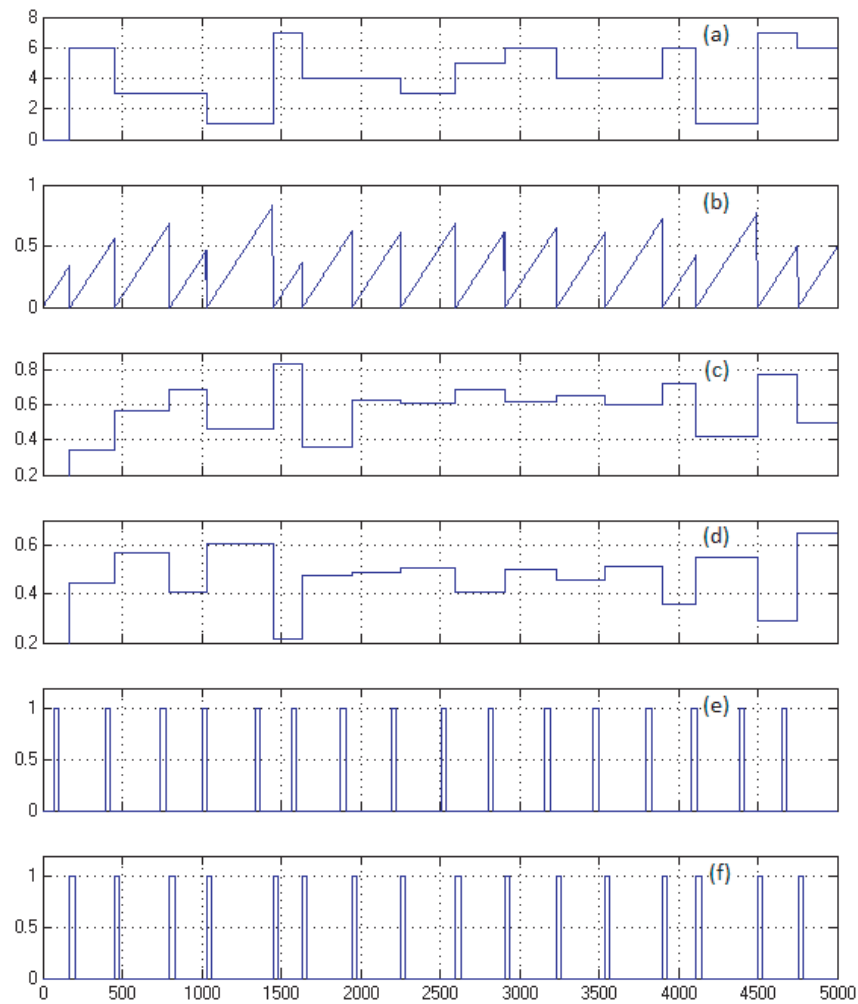

Figure 11. Time-domain signals of the 8-ary modulator. (a): the reference signal $S_{n} ;($ b): output of the counter $C(t)$; (c) and (d): input and output of the chaotic threshold generator $F(\cdot)$, respectively; (e): clock pulses (f): the output 8-ary CPPM signal.
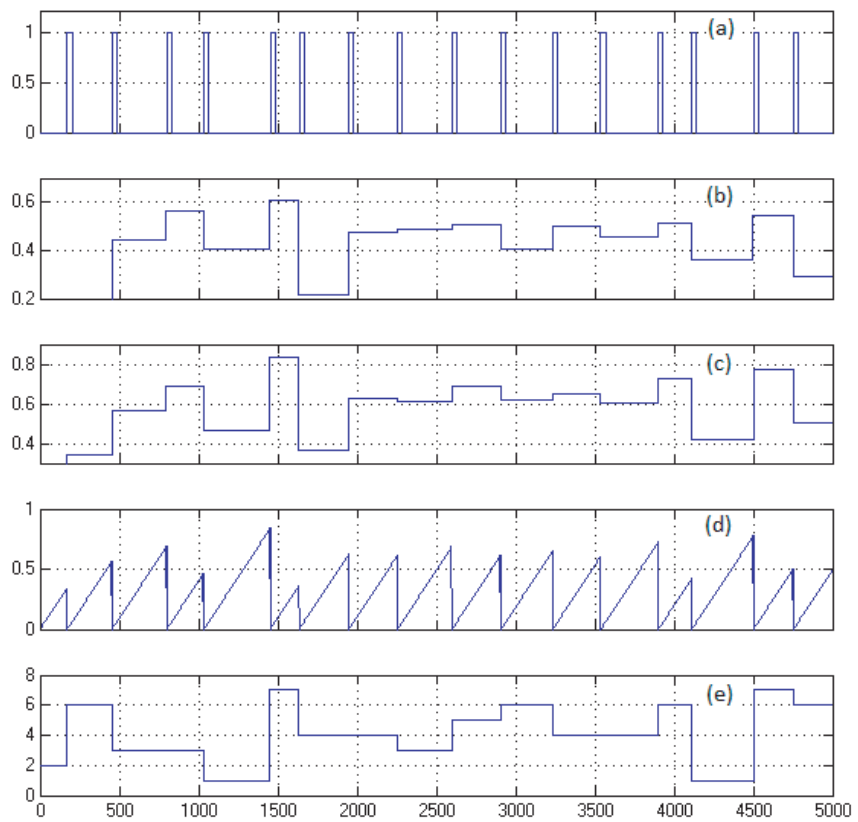

Figure 12. Time-domain signals of the 8-ary demodulator. (a): the input 8-ary CPPM signal; (b) and (c): outputs of the blocks S+H 2 and $\mathrm{S}+\mathrm{H} 1$, respectively; (d): output signal from the counter; (e): recovered signal $S_{n}$.

and demodulator circuits is $1 \mathrm{MHz}\left(10^{6}\right.$ pulses per second), for example, then the corresponding bit rates for $M=2,4,8$, and 16 are $3.749 \mathrm{Kbps}, 6.774 \mathrm{Kbps}$, $9.984 \mathrm{Kbps}$, and $13.136 \mathrm{Kbps}$, respectively. 


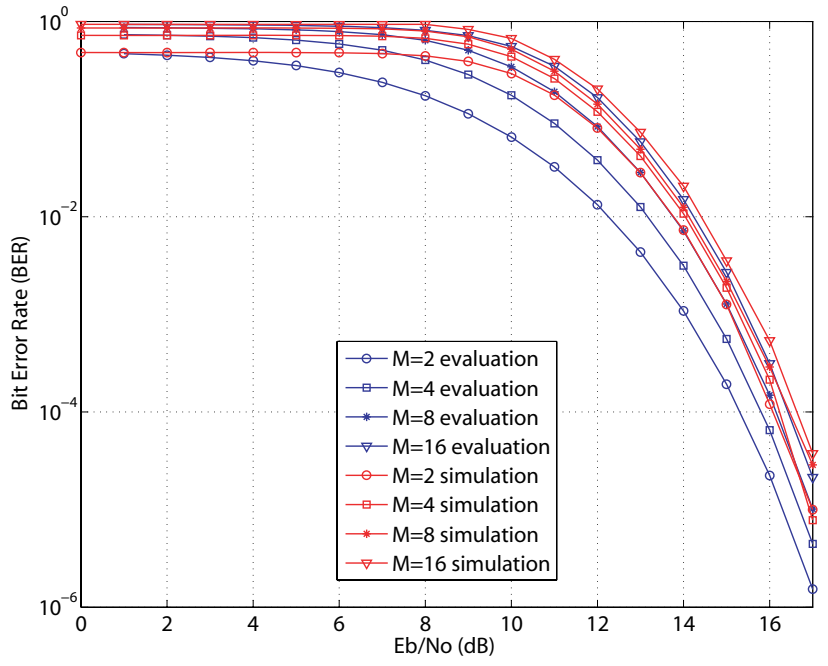

Figure 13. Theoretical vs. simulated BER results for the 2-, 4-, 8-, and 16-ary CPPM systems.

\subsection{BER Performance}

The BER performance obtained from simulations of the 2-, 4-, 8-, and 16-ary CPPM systems with a filtered AWGN channel is presented in Figure 13 (red lines). This BER is calculated as the number of error bits divided by the total number of transmitted bits in a time duration of $10^{8} \tau$. Similar to the theoretical curves, there are only slight differences between the simulation curves of the 2-, 4-, 8-, and 16-ary CPPM systems. Besides, with a same value of the fraction $E_{b} / N_{o}$, the simulation BER values are slightly higher than their corresponding theoretical ones. It can be explained that the cause of these differences is the loss of synchronization leading to bit errors. In theoretical evaluation, we suppose that the synchronization state is maintained at all times, thus pulse errors leading to bit errors can only occur due to noise and filtering. However, in the simulations, the effects of noise and filtering may cause not only pulse errors but also the loss of synchronization which leads to bit errors.

It can be observed from simulation results that, when the number of symbols, $M$, increases, the bit rate is improved significantly but the BER just slightly increases. This is a great advantage of the proposed $M$-ary CPPM method.

\section{CONCLUSIONS}

The paper has presented our development of the $M$ ary Chaotic Pulse Position Modulation technique aimed at improving the bit rate in secure communication systems. The proposed schemes for the modulator and demodulator of the system are designed and evaluated using both theoretical evaluation and numerical simulation in terms of time-domain signals and BER performance. In addition, the $M$-ary CPPM tent map is investigated considering the variation of the modulation parameters versus the chaotic behavior of the system. It can be seen from presented results that the $M$-ary CPPM not only inherits valuable features of the CPPM- based methods such as strong security and privacy due to its chaotic behavior, good performance in noise- and distortion-affected environments; but can also achieve a much higher bit rate with only a slightly different BER performance as compared to the conventional CPPM. This is the most important point to make the $M$-ary CPPM become a strong candidate for applications of chaos-based digital communication, especially in UWB and high security-required systems.

\section{ACKNOWLEDGMENT}

This work is carried out in the framework of the research project No.102.992010.17 supported by the Vietnam's National Foundation for Science and Technology Development (NAFOSTED). The authors would like to thank the NAFOSTED for supporting this work.

\section{REFERENCES}

[1] S. H. Strogatz, Nonlinear Dynamics And Chaos: With Applications To Physics, Biology, Chemistry, And Engineering. Westview Press, 2001.

[2] M. P. Kennedy and G. Kolumbán, "Special issue on "noncoherent chaotic communications"," IEEE Transactions on Circuits and Systems I: Fundamental Theory and Applications, vol. 47, no. 12, pp. 1661-1662, Dec. 2000.

[3] P. Stavroulakis, Ed., Chaos Applications in Telecommunications. CRC Press, 2005.

[4] W. M. Tam, F. C. M. Lau, and C. K. Tse, Digital Communications with Chaos: Multiple Access Techniques and Performance. Elsevier Science, 2006.

[5] L. M. Pecora and T. L. Carroll, "Synchronization in chaotic systems," Physical Review Letters, vol. 64, no. 8, pp. 821-824, Feb. 1990.

[6] N. F. Rulkov and L. S. Tsimring, "Synchronization methods for communication with chaos over band-limited channels," International Journal of Circuit Theory and Applications, vol. 27, no. 6, pp. 555-567, Nov./Dec. 1999.

[7] C.-C. Chen and K. Yao, "Numerical evaluation of error probabilities of self-synchronizing chaotic communications," IEEE Communications Letters, vol. 4, no. 2, pp. 3739, Feb. 2000

[8] N. F. Rulkov, M. M. Sushchik, L. S. Tsimring, and A. R. Volkovskii, "Digital communication using chaotic-pulseposition modulation," IEEE Transactions on Circuits and Systems I: Fundamental Theory and Applications, vol. 48, no. 12, pp. 1436-1444, Dec. 2001.

[9] J. Sushchik, M., N. Rulkov, L. Larson, L. Tsimrin, H. Abarbanel, K. Yao, and A. Volkovskii, "Chaotic pulse position modulation: A robust method of communicating with chaos," IEEE Communications Letters, vol. 4, no. 4, pp. 128-130, Apr. 2000.

[10] H. Torikai, T. Saito, and W. Schwarz, "Synchronization via multiplex pulse trains," IEEE Transactions on Circuits and Systems I: Fundamental Theory and Applications, vol. 46, no. 9, pp. 1072-1085, Sep. 1999.

[11] Y. Kim and et al., Chaotic UWB System, IEEE Std. 802.1505-0132-03-004a, Mar. 2005.

[12] P. Chiang and $\mathrm{C}$. $\mathrm{Hu}$, "Chaotic pulse-position baseband modulation for an ultra-wideband transceiver in CMOS," IEEE Transactions on Circuits and Systems II: Express Briefs, vol. 57, no. 8, pp. 642-646, Aug. 2010.

[13] J. T. Bean and P. J. Langlois, "A current mode analog circuit for tent maps using piecewise linear functions," in Proc. IEEE International Symp. Circuits and Systems (ISCAS'94), vol. 6, London, UK, 30 May - 2 June 1994, pp. $125-128$. 
[14] T. Addabbo, M. Alioto, A. Fort, S. Rocchi, and V. Vignoli, "The digital tent map: Performance analysis and optimized design as a low-complexity source of pseudorandom bits," IEEE Transactions on Instrumentation and Measurement, vol. 55, no. 5, pp. 1451-1458, Oct. 2006.

[15] H. Ruan, E. E. Yaz, T. Zhai, and Y. I. Yaz, "A generalization of tent map and its use in EKF based chaotic parameter modulation/demodulation," in Proc. 43rd IEEE Conf Decision and Control, vol. 2, 14-17 Dec. 2004, pp. 20712075.

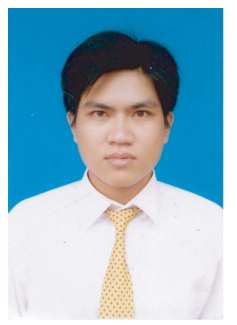

Nguyen Xuan Quyen was born in Nghe An, Viet Nam in 1983. He received the Diploma of Engineer and Master degree from the School of Electronics and Telecommunications (SET), Hanoi University of Science and Technology (HUST) in 2006 and 2008, respectively. From 2007 to 2011, he was a lecturer at the Department of Telecommunication Systems of SETHUST. Currently, he is working toward PhD degree at Institute for Smart System Technologies, Alpen-Adria University in Klagenfurt, Austria. His main research interests are microwave circuits, ultra wide band communications and chaos-based digital communications.

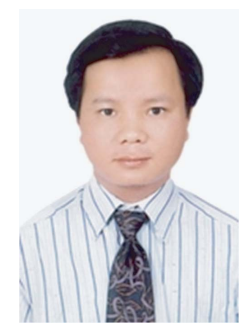

Vu Van Yem was born in 1975 in Hai Phong, Vietnam. He received the Ph.D. degree in communications from the Department of Electronics and Communications, TELECOM ParisTech (formerly ENST Paris) France in 2005. From 2006 to 2007, he was a postdoctoral researcher at the Department of Hyperfrequencies and Semiconductor, Institute of Electronics, Microelectronics and Nanotechnology (IEMN), France. He has been qualified to be named as Associate Professor since November, 2009. Currently, he is the head of the department of telecommunication systems, school of electronics and telecommunications, Hanoi University of Science and Technology, Vietnam. His areas of expertise are microwave engineering, antenna, chaos-based digital communications as well as wireless communication and localization systems.

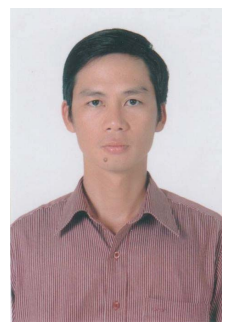

Thang Manh Hoang received the B.S. and M.S. degrees in Electronics and Telecommunications from Hanoi University of Science and Technology in 1998 and in 2001, respectively. During 1998-2004, he had stayed with Faculty of Electronics and Telecommunications, Hanoi University of Science and Technology. $\mathrm{He}$ received a PhD degree in Information and Control Engineering in 2007 at Nagaoka University of Technology, Japan. Now, he is a lecturer at Department of Electronics and Computer Engineering, School of Electronics and Telecommunications, Hanoi University of Science and Technology, Hanoi, Vietnam. $\mathrm{He}$ is an Executive Member of IEEE Vietnam Section and of Korea Information and Communications Society (KICS), a member of IEEE, IEEE Circuits and Systems Society (CAS), IEEE Communications Society (ComSoc), Korea Information and Communications Society (KICS), Radio Electronics Vietnam (REV). 